\title{
Rhododendrons in Indian Himalayan Region: Diversity and Conservation
}

\author{
K. Chandra Sekar ${ }^{1}$, Sunil Kumar Srivastava ${ }^{2}$ \\ ${ }^{1}$ G.B. Pant Institute of Himalayan Environment \& Development, Kosi, Almora, Uttarakhand, India; ${ }^{2}$ Botanical Survey of India, \\ Northern Circle, Dehradun, India. \\ Email: "kcsekar1312@rediffmail.com
}

Received July 23 ${ }^{\text {rd }}$, 2010; revised October $27^{\text {th }}, 2010$; accepted November $2^{\text {nd }}, 2010$.

\begin{abstract}
The genus Rhododendron of Indian Himalayan Region (IHR) has been enumerated in the present paper. A total of 87 species, 12 subspecies and 8 varieties of Rhododendrons recorded in IHR, among these 6 species and one subspecies are reported from Western Himalaya. The maximum concentration of 86\% observed in Arunachal Pradesh (75 species). The species of Rhododendrons exhibit significant diversity in habit and broad range of distribution from the altitude of 800-6000 m. and the best range is observed in 3001-3500 m altitudes. In analysis revealed 20 taxa are endemic, 30 are rare, 24 are threatened / endangered, 3 are vulnerable and 47 taxa have to be assessed. The major threats to rhododendrons are deforestation and unsustainable extraction for firewood and incense by local people has been discussed.
\end{abstract}

Keywords: Rhododendrons, Indian Himalayan Region, Ericaceae, India

\section{Introduction}

The genus Rhododendron, family Ericaceae, was founded by Linnaeus [1]. The word Rhododendron is derived from two Greek words rhodon (rose) and dendron (tree) meaning rose tree. The genus with attractive and beautyful flowers is represented by 850 species in the world [2]. They are mostly distributed at higher elevations in the Sino-Himalayan region with maximum concentration in Western China [3]. In India, the species are mostly confined to the Himalayan region, particularly in Eastern Himalaya. A revision of the genus was carried out by Cullen [4], Chamberlain [5], Philipson and Philipson [6], Chamberlain and Rae [7], Kron [8] and, Judd and Kron [9]. Preliminary enumerations and inventories of the genus were made by Pradhan [3,10], Ghosh and Samaddar [11], Bhattacharyya and Sanjappa [12]. Sastry and Hajra [13] and Mao et al. [14] were made the contribution on rare and endemic Rhododendrons of India. The Rhododendrons of Sikkim-Himalaya region were done by Pradhan and Lachungpa [15] and Singh et al. [16]. The importance of Rhododendrons in Meghalaya dealt by Yumnam [17].

The Indian Himalayan Region (IHR) occupies a special place in the mountain ecosystems of the world. The IHR is one of the most fragile mountain regions of the world and holds an enormous repository of biological diversity which is increasingly under pressure from human activities. The region comprises a rich variety of flora, fauna, human communities and culture. Of the estimated 8,000 species of vascular plants in the Himalayan region, around 3,160 are endemic and 450 species are endangered $[18,19]$. The indiscriminate exploitation, destruction of habitats, spread of harmful chemicals and introduction of alien species, a number of plants have been disappeared while others await a smilar fate [20]. As a result, gap between demand and supply is widening. In this regards, global efforts are being made to conserve the phytodiversity especially rare, endangered and threatened species, which are known to be important component of biodiversity.

Due to human interference the natural populations of rhododendrons in the entire Himalaya are gradually diminishing. The major threats to rhododendrons are deforestation and unsustainable extraction for firewood and incense by local people. A set of rhododendrons which are classified as rare/endangered may be wiped out from the biota in the near future if proper conservation measures are not made. So, the present task of diversity and conservation status of Rhododendrons of Indian Himalayan Region has made.

\section{Methodology}

The present work on Rhododendrons of Indian Himalaya 
is based on extensive literature surveys made in different states of Indian Himalayan Region (IHR). For the threat categories, we have consulted different published scientific papers, monographs, red-list documents, IUCN list, etc. All the taxa have been listed alphabetically with altitude, distribution in Indian Himalayan States and other region, and revealed threat categories. For enumeration of species, the IHR is divided into two botanical regions, namely the Western Himlaya and the Eastern Himalaya. The Western Himalaya region is the states of Jammu \& Kashmir, Himachal Pradesh and Uttaranchal. Similarly, the Eastern Himalayan region includes the seven sisters states of North-Eastern India (Arunachal Pradesh, Assam, Manipur, Meghalaya, Mizoram, Nagaland and Tripura), Sikkim and Darjeeling district of West Bengal. The species of Rhododendrons are calculated and enumerated in state wise representation of Western and Eastern Himalaya.

\section{Results}

A total of 87 species, 12 subspecies and 8 varieties of Rhododendrons recorded in IHR (Table 1). The Western Himalaya has 6 species, namely Rhododendron anthopogon (Plate 1a), R. arboreum (Plate 1b), R. barbatum, $R$. campanulatum (Plate 1c), R. lepidotum and R. nivale; while Eastern Himalaya is represented by all enumerated species. The maximum concentration of species is observed in Arunachal Pradesh (86\%). Out of 87 species known from IHR, 75 species occur in the state of Arunachal Pradesh alone. The state wise distribution of species is showed in Figure 1. The distribution of species in relation to altitude is shown in Figure 2. The maximum numbers of Rhododenrons are present in the 3001-3500 $\mathrm{m}$ altitudes, lowest in 500-1000 $\mathrm{m}$ and above $5000 \mathrm{~m}$, and absent in less than $500 \mathrm{~m}$ (Figure 2). A single species namely $R$. arboreum $\mathrm{Sm}$. is only found in less then $1000 \mathrm{~m}$ altitude (from $800 \mathrm{~m}$ onwards) and found in almost all the states of IHR except Assam and Tripura. $R$. nivale Hook. f. is a single species found in above $5000 \mathrm{~m}$ altitude and found in the state of Sikkim and Uttarakhand. The taxa endemic to Arunachal Pradesh are R. arboreum Sm. subsp. delavayi (Franch.) D. F. Chamb., R. chamaethomsonii (Tagg) Cowan \& Davidian, $R$. concin- noides Hutch. \& Kingdon-Ward, R.. falconeri Hook. f. subsp. eximium (Nattau) D. F. Chamb., $R$. imberbe Hutch., $R$. nayarii G.D. Pal, R. santapaui Sastry, Kataki, P. Cox, Patricia Cox \& P. Hutch. and R. subansiriense D. F. Chamb. \& Cox. The taxa namely $R$. candelabrum Hook. f. and $R$. sikkimense U. C. Pradhan \& S. T. Lachungpa are endemic to Sikkim. $R$. formosum Kingdon-Ward is endemic to the states of Arunachal Pradesh and Meghalaya. A variety, R. triflorum Hook var. bauhiniiflorum (Watt ex Hutch.) J. Cullen is endemic to Manipur and categorized as Rare in distribution. $R$. decipiens Lacait. is endemic to Sikkim and West Bengal hills. A variety namely, $R$. formosum Wall. var. inaequale (Hutch.) J. Cullen is endemic to Meghalaya, Mizoram and Nagaland. The species $R$. johnstoneanum Watt ex Hutch. is endemic and endangered in Arunachal Pradesh, Manipur and Mizoram. R. macabeanum Watt ex Balf. f. is endemic and found in rare in Manipur and Nagaland.

Table 1. Rhododendrons of Indian Himalayan Region.

\begin{tabular}{|c|c|c|c|c|c|}
\hline \multirow{2}{*}{ S. No. } & \multirow{2}{*}{ NAME OF THE TAXA } & \multirow{2}{*}{ ALTITUDE (IN METRE) } & \multicolumn{2}{|c|}{ DISTRIBUTION } & \multirow{2}{*}{ STATUS } \\
\hline & & & India & Other region & \\
\hline 1 & R. anthopogon D. Don & $3350-5000$ & AP, HP, SK, WB & Bhutan, China, Nepal, Tibet & $\mathrm{NE}$ \\
\hline 3 & R. arboreum Sm. & $800-3000$ & $\begin{array}{l}\text { AP, HP, JK, UK, MN, } \\
\text { MG, MZ, NG, SK, WB }\end{array}$ & $\begin{array}{c}\text { Bhutan, Myanmar, Nepal, Sri } \\
\text { Lanka, Pakistan, Tibet }\end{array}$ & $\mathrm{NE}$ \\
\hline 4 & $\begin{array}{l}\text { R. arboreum Sm. subsp. } \\
\text { Cinnamomeum } \\
\text { (Wall. ex G. Don) Tagg }\end{array}$ & c. 2500 & SK, WB & Nepal & $\mathrm{NE}$ \\
\hline 5 & R. arboreum Sm. var. roseum Lindl. & $2500-3600$ & SK, WB & Bhutan, China, Myanmar & $\mathrm{NE}$ \\
\hline 6 & $\begin{array}{l}\text { R. arboreum Sm. subsp. } \\
\text { delavayi (Franch.) } \\
\text { D. F. Chamb. }\end{array}$ & $2500-3200$ & AP, MN, MG & China, Myanmar, Thailand & $\mathrm{NE}$ \\
\hline 7 & $\begin{array}{l}\text { R. arboreum var. peramoenum (Balf. } \\
\begin{array}{ll}\text { f. \& Forrest) } & \text { D.F. Chamb. }\end{array}\end{array}$ & $3000-3200$ & $\mathrm{AP}$ & - & $\mathrm{EN}^{1}$ \\
\hline 8 & R. assamicum Kingdon-Ward & c. 3000 & AP & - & $\mathrm{EN}^{1}$ \\
\hline 10 & R. barbatum G. Don & $2500-3700$ & AP, SK, UK, WB & Bhutan, China, Nepal & NE \\
\hline 11 & R. beanianum Cowan & $3000-3350$ & AP & Myanmar & $\mathrm{RA}^{1}$ \\
\hline 12 & R. boothii Nutt. & $1800-2500$ & AP & Bhutan, China & $\mathrm{TN}^{1}$ \\
\hline 13 & R. bulu Hutch. & $3000-3800$ & AP & China, Tibet & $\mathrm{TN}^{1}$ \\
\hline 14 & $\begin{array}{l}\text { R. calostrotum Balf. f. \& King- } \\
\text { don-Ward subsp. riparium (King- } \\
\text { don-Ward) J. Cullen }\end{array}$ & $3000-4500$ & AP & China, Myanmar & $\mathrm{RA}^{1}$ \\
\hline
\end{tabular}


Continued Table 1

\begin{tabular}{|c|c|c|c|c|c|}
\hline 15 & R. camelliaeflorum Hook. f. & $2700-4000$ & AP, SK & China, Nepal, Tibet & $\mathrm{NE}$ \\
\hline 16 & $\begin{array}{ll}\text { R. campanulatum } & \text { D. Don }\end{array}$ & $2500-4300$ & $\begin{array}{l}\text { AP, HP, JK, SK, } \\
\text { UK, WB }\end{array}$ & Bhutan, Nepal & $\mathrm{NE}$ \\
\hline 17 & $\begin{array}{l}\text { R. campanulatum D. Don subsp. } \\
\text { aeruginosum (Hook. f.) } \\
\text { D. F. } \\
\text { Chamb. }\end{array}$ & $4500-5000$ & SK & Bhutan, Nepal & $\mathrm{NE}$ \\
\hline 18 & $\begin{array}{l}\text { R. campanulatum D. Don var. } \\
\text { wallichii Hook. f. }\end{array}$ & 4000 & AP, SK & Bhutan, Nepal & $\mathrm{NE}$ \\
\hline 19 & R. campylocarpum Hook. f. & $3300-4300$ & AP, SK & $\begin{array}{c}\text { Bhutan, Myanmar, Nepal, } \\
\text { Tibet }\end{array}$ & $\mathrm{RA}^{1}$ \\
\hline 20 & R. campylogynum Franch. & $2700-4300$ & AP & China, Myanmar & $\mathrm{NE}$ \\
\hline 21 & R. candelabrum Hook. f. & $3600-4300$ & SK & - & $\mathrm{EN}^{1}$ \\
\hline 22 & R. cephalanthum Franch. & $3000-4500$ & $\mathrm{AP}$ & China, Myanmar & $\mathrm{RA}^{1}$ \\
\hline 23 & R. cerasinum Tagg & $3000-3200$ & AP & China, Myanmar, Tibet & $\mathrm{NE}$ \\
\hline 24 & $\begin{array}{c}\text { R. chamaethomsonii (Tagg) Cowan \& } \\
\text { Davidian }\end{array}$ & $3600-5000$ & AP & - & $\mathrm{EN}^{1}$ \\
\hline 25 & R. ciliatum Hook. f. & $2700-3400$ & SK & Bhutan, China, Nepal & $\mathrm{NE}$ \\
\hline 26 & R. cinnabarinum Hook. f. & $3000-4000$ & AP & Bhutan, China, Nepal, Tibet & $\mathrm{NE}$ \\
\hline 27 & $\begin{array}{l}\text { R. cinnabarinum Hook. f. subsp. } \\
\text { xanthocodon } \quad \text { (Hutch.) J. Cullen }\end{array}$ & $3000-4000$ & AP & Bhutan, China & $\mathrm{TN}^{1}$ \\
\hline 28 & $\begin{array}{l}\text { R. concinnoides Hutch. \& } \\
\text { Kingdon-Ward }\end{array}$ & $2400-3400$ & AP & - & $\mathrm{EN}^{1}, \mathrm{TN}^{1}$ \\
\hline 29 & R. coxianum Davidian & c. 1800 & AP & - & $\mathrm{EN}^{1}$ \\
\hline 30 & R. crinigerum Franch. & $3100-4000$ & $\mathrm{AP}$ & China, Myanmar & $\mathrm{NE}$ \\
\hline 31 & R. dalhousiae Hook. f. & $1800-2300$ & AP, SK, WB & Bhutan, Nepal & $\mathrm{NE}$ \\
\hline 32 & $\begin{array}{l}\text { rhabdotum (Balf. f. \& Cooper) } \\
\text { J. Cullen }\end{array}$ & c. 2500 & AP & Bhutan, China & $\mathrm{RA}^{1}, \mathrm{VU}^{3}$ \\
\hline 33 & R. decipiens Lacait. & 2500-3000 & SK, WB & - & $\mathrm{EN}^{1}$ \\
\hline 34 & $R$. dendricola Hutch. & $1200-1400$ & AP & China, Myanmar & $\mathrm{RA}^{1}$ \\
\hline 35 & R. edgeworthii Hook. f. & $2100-3300$ & $\mathrm{AP}, \mathrm{SK}$ & Bhutan, China, Myanmar & $\mathrm{RA}^{1}$ \\
\hline 36 & R. elliottii Watt & $2700-3000$ & $\mathrm{MN}, \mathrm{NG}$ & - & $\mathrm{EN}^{1}, \mathrm{ED}^{1}$ \\
\hline 37 & R. eudoxum Balf. f. \& Forrest & $3300-4000$ & AP & China, Tibet & $\mathrm{NE}$ \\
\hline 38 & $\begin{array}{l}\text { R. eudoxum Balf. f. \& Forrest subsp. } \\
\text { tamenium (Balf. f. \& Forrest) Tagg }\end{array}$ & $3300-4000$ & AP & Bhutan, China & $\mathrm{NE}$ \\
\hline 39 & R. exasperatum Tagg & $3000-4000$ & AP & China, Myanmar & $\mathrm{RA}^{1}$ \\
\hline 40 & R. falconeri Hook. f. & $2100-4000$ & AP, SK, WB & Bhutan, Nepal & $\mathrm{NE}$ \\
\hline 41 & $\begin{array}{l}\text { R. falconeri Hook. f. subsp. eximium } \\
\text { (Nattau) D. F. Chamb. }\end{array}$ & $3000-3500$ & AP & - & $\mathrm{EN}^{1}, \mathrm{ED}^{1}$ \\
\hline 42 & R. formosum Kingdon-Ward & $1500-2000$ & MG & - & $\mathrm{EN}^{1}, \mathrm{TN}^{1}$ \\
\hline 43 & $\begin{array}{l}\text { R. formosum Wall. var. inaequale } \\
\text { (Hutch.) J. Cullen }\end{array}$ & $1500-2000$ & MG, MZ, NG & - & $\mathrm{EN}^{1}, \mathrm{TN}^{1}$ \\
\hline 44 & R. fulgens Hook. f. & $3000-4300$ & AP, SK, WB & Bhutan, China, Nepal, Tibet & $\mathrm{RA}^{2}$ \\
\hline 45 & R. fulvum Balf. f. \& W. W. Smith & 2460-3385 & $\mathrm{AP}$ & China, Myanmar, Tibet & $\mathrm{NE}$ \\
\hline 46 & R. glaucophyllum Rehder & $3080-3700$ & SK & Bhutan, Nepal, Tibet & $\mathrm{NE}$ \\
\hline 47 & $\begin{array}{l}\text { R. glaucophyllum Rehder var. } \\
\text { tubiforme Cowan \& Davidian }\end{array}$ & 3100 & AP & Bhutan, China, Myanmar & $\mathrm{NE}$ \\
\hline 48 & R. grande Wight & 2160-3385 & $\mathrm{AP}, \mathrm{SK}, \mathrm{WB}$ & Bhutan, China, Napel, Tibet & $\mathrm{NE}$ \\
\hline 49 & R. griffithianum Wight & $2160-2770$ & AP, SK, WB & Bhutan, Napal, Tibet & $\mathrm{NE}$ \\
\hline 50 & R. hodgsonii Hook. f. & $3080-3690$ & AP, SK,WB & Bhutan, Napal, Tibet & $\mathrm{NE}$ \\
\hline 51 & R. hookeri Nutt. & $2500-3700$ & AP & Bhutan & $\mathrm{RA}^{1}$ \\
\hline 52 & R. imberbe Hutch. & 2770 & $\mathrm{AP}$ & - & $\mathrm{EN}^{1}$ \\
\hline 53 & R. johnstoneanum Watt ex Hutch. & $1160-3000$ & AP, MN, MZ & - & $\mathrm{EN}^{1}, \mathrm{ED}^{1}$ \\
\hline 54 & R. kasoense Hutch. \& Kingdon-Ward & $2500-2700$ & AP & China & $\mathrm{RA}^{1}$ \\
\hline 55 & R. kendrickii Nutt. & $2300-2800$ & AP & Phutan, China & $\mathrm{RA}^{1}$ \\
\hline 56 & R. keysii Nutt. & $2440-3650$ & $\mathrm{AP}, \mathrm{SK}$ & Bhutan, China, Tibet & $\mathrm{RA}^{1}$ \\
\hline 57 & R. lanatum Hook. f. & $3080-4000$ & $\mathrm{AP}, \mathrm{SK}$ & Bhutan, China, Tibet & $\mathrm{NE}$ \\
\hline 58 & R. lanigerum Tagg & 3080-3385 & AP & China, Tibet & $\mathrm{NE}$ \\
\hline 59 & R. lepidotum Wall. ex D. Don & $2160-4620$ & AP, JK, HP, SK,UK & $\begin{array}{l}\text { Bhutan, China, Myanmar, } \\
\text { Napal, Pakistan }\end{array}$ & $\mathrm{NE}$ \\
\hline 60 & R. leptocarpum Nutt. & $2300-4310$ & $\mathrm{AP}, \mathrm{SK}$ & $\begin{array}{c}\text { Bhutan, China, Myanmar, } \\
\text { Tybet }\end{array}$ & $\mathrm{ED}^{2}$ \\
\hline 61 & R. lindleyi T. Moore & $1850-3080$ & AP, MN, SK,WB & $\begin{array}{c}\text { Bhutan, China, Myanmar, } \\
\text { Napal, Tibet }\end{array}$ & $\mathrm{NE}$ \\
\hline 62 & R. macabeanum Watt ex Balf. f. & 2500-3000 & $\mathrm{MN}, \mathrm{NG}$ & - & $\mathrm{EN}^{1}, \mathrm{RA}^{1}$ \\
\hline 63 & $\begin{array}{l}\text { R. maddenii Hook. f. subsp. crassum } \\
\text { (Franch.) J. Cullen }\end{array}$ & $2250-3000$ & MN, MG, NG & $\begin{array}{c}\text { Bhutan, China, Myanmar, } \\
\text { Vietnam }\end{array}$ & $\mathrm{RA}^{1}$ \\
\hline
\end{tabular}


Continued Table 1

\begin{tabular}{|c|c|c|c|c|c|}
\hline 64 & R. maddenii Hook. f. & $2400-3650$ & $\mathrm{AP}, \mathrm{SK}$ & Bhutan, China & $\mathrm{RA}^{1}, \mathrm{ED}^{2}$ \\
\hline 65 & $\begin{array}{l}\text { R. megacalyx Balf. f. \& } \\
\text { Kingdon-Ward }\end{array}$ & $2160-2770$ & AP & China, Myanmar & $\mathrm{RA}^{1}$ \\
\hline 66 & $\begin{array}{l}\text { R. megeratum Balf. f. \& Forrest } \\
\text { R. mekongense Franch. var. }\end{array}$ & $3050-4150$ & AP & China, Myanmar, Tibet & $\mathrm{RA}^{1}$ \\
\hline 67 & $\begin{array}{l}\text { rubrolineatum (Balf. f. \& Forr.) } \\
\text { J. Cullen }\end{array}$ & $3350-4250$ & AP & China & $\mathrm{RA}^{1}$ \\
\hline 68 & $\begin{array}{c}\text { R. nayarii G.D. Pal } \\
\text { R. neriiflorum Franch. subsp. }\end{array}$ & $2500-3000$ & AP & - & NE \\
\hline 69 & $\begin{array}{c}\text { phaedropum } \\
\text { (Balf. f. \& Farrer) Tagg }\end{array}$ & 3000 & AP & Bhutan, China, Myanmar & $\mathrm{TN}^{1}$ \\
\hline 70 & R. nivale Hook. f. & $4000-6000$ & SK, UK & Butan, China, Nepal & NE \\
\hline 71 & R. niveum Hook. f. & $3080-3700$ & $\mathrm{AP}, \mathrm{SK}$ & Bhutan & $\mathrm{ED}^{2}$ \\
\hline 72 & R. nuttallii Booth ex Nutt. & $1200-3650$ & AP & Bhutan, China, Myanmar & $\mathrm{RA}^{1}$ \\
\hline 73 & R. obtusum Hort. ex Wats & 1500 & AP & Japan, Myanmar & NE \\
\hline 74 & R. papillatum Balf. f. \& Copper & $1800-3300$ & AP, SK & Bhutan, Nepal & $\mathrm{RA}^{1}$ \\
\hline 75 & R. pemakoense Kingdon-Ward & $2400-3050$ & AP & China & $\mathrm{RA}^{1}$ \\
\hline 76 & R. pendulum Hook. f. & $2270-3650$ & AP, SK & Bhutan, China, Nepal, Tibet & $\mathrm{RA}^{1}$ \\
\hline 77 & R. pocophorum Balf. f. ex Tagg & $3650-4600$ & AP & China & $\mathrm{RA}^{1}$ \\
\hline 78 & $\begin{array}{l}\text { R. pruniflorum Hutch. \& } \\
\text { Kingdon-Ward }\end{array}$ & 3050-3950 & AP & Myanmar & $\mathrm{RA}^{1}$ \\
\hline 79 & R. pumilum Hook. $\mathrm{f}$. & $3500-4500$ & AP, SK & $\begin{array}{l}\text { Bhutan, China, Myanmar, } \\
\text { Nepal, Tibet }\end{array}$ & $\mathrm{ED}^{2}$ \\
\hline 80 & $\begin{array}{l}\text { R. rex Levl. subsp. Arizelum } \\
\text { (Balf. f. \& Forr.) D. F. Chamb. }\end{array}$ & $3000-4000$ & AP & China, Myanmar & $\mathrm{RA}^{1}$ \\
\hline 81 & $\begin{array}{l}\text { R. santapaui Sastry, Kataki, } \\
\text { P. Cox, Patricia Cox \& P. Hutch. }\end{array}$ & 2300 & AP & - & $\mathrm{EN}^{1}, \mathrm{ED}^{1}$ \\
\hline 82 & R. setosum D. Don & $2160-4950$ & AP, SK, WB & Bhutan, China, Nepal, Tibet & NE \\
\hline 83 & $R$. sidereum Balf. f. & $2770-3080$ & $\mathrm{AP}$ & China, Myanmar & NE \\
\hline 84 & $\begin{array}{l}\text { R. sikkimense U. C. Pradhan \& S. T. } \\
\text { Lachungpa }\end{array}$ & 3700 & SK & - & $\mathrm{EN}^{1}$ \\
\hline 85 & R. sinogrande Balf. f. & $3080-4310$ & AP & China, Myanmar, Tibet & $\mathrm{NE}$ \\
\hline 86 & R. smithii Nutt. & $2160-3700$ & AP, SK & Bhutan, China & $\mathrm{NE}$ \\
\hline 87 & R. stenaulum Balf. f. \& W. W. Smith & 2770 & AP & China & $\mathrm{NE}$ \\
\hline 88 & R. stewartianum Diels & $3080-4310$ & AP & China, Myanmar, Tibet & NE \\
\hline 89 & R. subansiriense D. F. Chamb. \& Cox & $2600-2800$ & AP & - & $\begin{array}{l}\mathrm{EN}^{1}, \mathrm{ED}^{1}, \\
\mathrm{VU}^{3}\end{array}$ \\
\hline 90 & R. succothii Davidian & $3400-4200$ & AP & Bhutan & $\mathrm{RA}^{1}$ \\
\hline 91 & R. taggianum Hutch. & $2160-3390$ & AP & China, Myanmar & $\mathrm{NE}$ \\
\hline 92 & $\begin{array}{l}\text { R. tanastylum Balf. f. \& } \\
\text { Kingdon-Ward }\end{array}$ & $1850-3350$ & AP & China, Myanmar & $\mathrm{RA}^{1}$ \\
\hline 93 & R. tephropeplum Balf. f. \& Farrer & $2450-4300$ & AP & China, Myanmar & $\mathrm{RA}^{1}$ \\
\hline 94 & R. thomsonii Hook. f. & $3390-4000$ & AP, NG, SK WB & Bhutan, Napal, Tibet & $\mathrm{NE}$ \\
\hline 95 & R. triflorum Hook. f. & $2160-2930$ & AP, SK, WB & $\begin{array}{l}\text { Bhutan, China, Myanmar, } \\
\text { Nepal, Tibet }\end{array}$ & $\mathrm{NE}$ \\
\hline 96 & $\begin{array}{c}\text { R. triflorum Hook var. } \\
\text { bauhiniiflorum (Watt ex Hutch.) } \\
\text { J. Cullen }\end{array}$ & $2470-3080$ & $\mathrm{MN}$ & - & $\mathrm{EN}^{1}, \mathrm{RA}^{1}$ \\
\hline 97 & R. tsariense Cowan & $2000-3000$ & AP & Bhutan, China & $\mathrm{NE}$ \\
\hline 98 & R. uvarifolium Diels & $2160-2470$ & $\mathrm{AP}$ & China & $\mathrm{NE}$ \\
\hline 99 & R. vaccinioides Hook. f. & $1850-3700$ & AP, SK, WB & $\begin{array}{c}\text { Bhutan, Myanmar, Napal, } \\
\text { Tibet }\end{array}$ & $\mathrm{NE}$ \\
\hline 100 & R. virgatum Hook. $\mathrm{f}$. & $2160-2770$ & AP, SK & Bhutan,China, Myanmar & NE \\
\hline 101 & $\begin{array}{l}R \text {. virgatum Hook. f. subsp. } \\
\text { oleifolium (Franchet) J. Cullen }\end{array}$ & $2200-3000$ & AP & China, Tibet & $\mathrm{NE}$ \\
\hline $\begin{array}{l}102 \\
103\end{array}$ & $\begin{array}{l}\text { R. veitchianum Hook. } \\
\text { R. wallichii Hook. f. }\end{array}$ & $\begin{array}{l}1230-1700 \\
4000-4500\end{array}$ & $\begin{array}{c}\text { MZ } \\
\text { SK, WB }\end{array}$ & $\begin{array}{l}\text { Loas, Myanmar, Thailand } \\
\text { Bhutan, China, Nepal }\end{array}$ & $\begin{array}{l}\mathrm{RA}^{1} \\
\mathrm{NE}\end{array}$ \\
\hline 104 & R. walongense Kingdon-Ward & $1500-2150$ & $\mathrm{AP}$ & China & $\mathrm{RA}^{1}$ \\
\hline 105 & R. wattii Cowan & 2700 & AP, MN & - & $\begin{array}{l}\mathrm{EN}^{1}, \mathrm{ED}^{1}, \\
\mathrm{VU}^{3}\end{array}$ \\
\hline 106 & R. wightii Hook. f. & $3050-4310$ & AP, SK & $\begin{array}{l}\text { Bhutan, China, Myanmar, } \\
\text { Nepal, Tibet }\end{array}$ & $\mathrm{RA}^{2}$ \\
\hline 107 & R. xanthostephanum Merrill & $1500-3000$ & AP, SK & China, Myanmar & $\mathrm{RA}^{1}$ \\
\hline
\end{tabular}

1 - Mao et al., 2002; 2 - Singh et al., 2003; 3 - Anonymous, 2009; AP - Arunachal Pradesh; ED - Endangered; EN - Endemic; HP - Himachal Pradesh; JK-Jammu \& Kashmir; MG - Meghalaya; MN - Manipur; MZ- Mizoram; NE - Not Evaluated; NG - Nagaland; RA - Rare; SK - Sikkim; TN-Threatened; UK - Uttarakhand; VU - Vulnerable; WB - West Bengal 


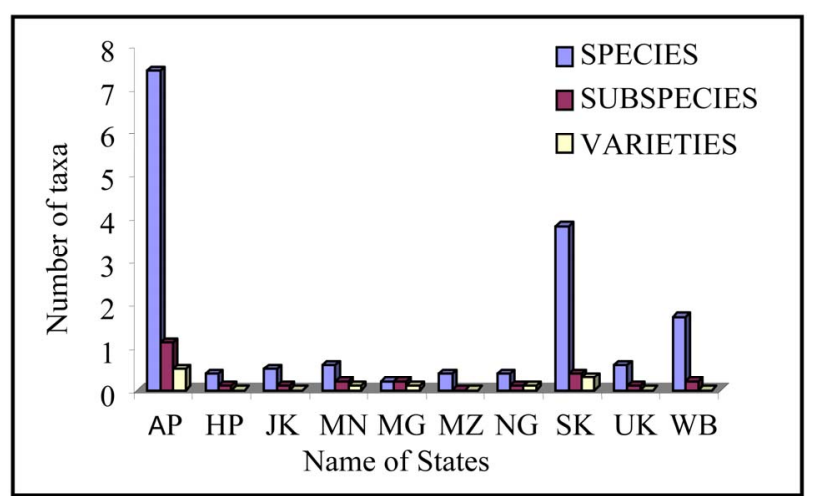

Name of the States: AP - Arunachal Pradesh; HP - Himachal Pradesh; JK-Jammu \& Kashmir; MG - Meghalaya; MN - Manipur; MZ- Mizoram; NG - Nagaland; SK - Sikkim; UK - Uttarakhand; WB - West Bengal

Figure 1. Distribution of rhododendrons in different states of IHR.

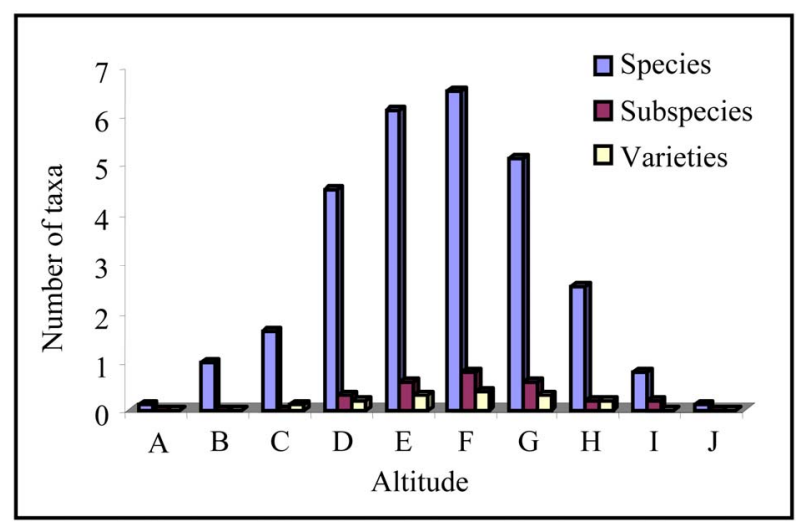

Altitudes (in m) A: 500-1000; B: 1000-1500; C: 1500-2000; D: 2000-2500; E: 2500-3000; F: 3000-3500; G: 3500-4000; H: 4000-4500; I: 4500-5000; J: $>5000$

Figure 2. Altitudinal diversity of rhododendrons in IHR.

\section{Discussion}

The species of Rhododendrons exhibit significant diversity in habit and broad range of distribution from the altitude of $800-6000 \mathrm{~m}$. A total of 87 species, 12 subspecies and 8 varieties of Rhododendrons recorded in IHR. Out of these species, 20 taxa are endemic to IHR. While considering the status, 47 taxa are not yet evaluated, 30 are rare, 24 are threatened / endangered and 3 are vulnerable [14,16,21]. In comparison with neighboring countries, China is having a total of 571 species of Rhododendrons, out of which 409 species are endemic [22] and other countries like Pakistan, Bhutan, Nepal, etc are having very less species diversity in Rhododendrons. A lot of exploration is still lacking to discover the species described in other parts of the country, because, the China and India is having similar habitat in support the growth of Rhododendrons. While considering the importance of altitude the maximum number of Rhododen- drons present in the altitudes of 3001-3500 m. These altitudes are considered as best suitable sites for Rhododendrons for conservation and multiplication.

In the recent days, IHR is greatly affected due to various threat posed by the nature as well as by human beings. Since Rhododendrons are the inhabitants of the IHR, they are also greatly affected and their population in the nature is gradually dwindling [14]. The rise in population with demand on land for farming, increased animal husbandry practices, construction of roadways, hydel-power stations and allied works, army personnel garrisoned at alpine locations and lately the tourist influx have collectively resulted in the building up of considerable pressure on the availability of rhododendron species. The major threats to rhododendrons are deforestation and unsustainable extraction for firewood and incense by local people. Due to the presence of polyphenols and flavonoids, rhododendrons make excellent firewood that burns even under wet conditions. Rhododendron firewood is also being used in the high-altitude trekking corridor for the purpose of tourism. Some of the species have already become scarce, for example, $R$. leptocarpum is endangered and reported to have only 16 surviving individuals at present in the Sikkim [16].

The conservation of Rhododendron species can be effected by two well established means, the in-situ and exsitu methods. In-situ conservation can be brought about by establishing Rhododendron sanctuaries, Parks, etc. Some efforts by Sikkim forest department and Sikkim Rhododendron Society have been made by fencing the Rhododendron rich sites and declaring them as Rhododendron Sanctuary between Lachung and Yumthang in the State. Similar efforts need to be made by Arunachal Pradesh Government as the state is home for more than 50 percent rare and endemic IHR species. The ex-situ conservation can be effected by cultivating Rhododendron species in the gardens and parks under suitable climatic conditions or by using tissue culture techniques. There should not be many difficulties in introducing these species in Botanic Gardens and Parks as most of them have successfully been introduced and cultivated in the European and American countries. The species of Rhododendron arboreum are propagated through cuttings $[23,24]$. Tissue culture studies of Indian Rhododendrons are recently initiated; only few species especially $R h o$ dodendron maddeni has only been propagated through tissue culture methods (Singh and Gurung, 2009) and some others are under progress. But in foreign countries, the Rhododendrons have already been carried out for commercial cultivation [26-32]. The standard culture medium for tissue culture methods is readily available in the market [14]. Successful tissue culture of these species will be a great contribution for rapid multiplication and 
towards in-vitro conservation. The success of conservation programme depends on the awareness of local people. It is imperative to educate the local inhabitants about the wealth of Rhododendrons and importance towards the conservation of biodiversity in IHR.

\section{Acknowledgements}

The authors are thankful to Dr. L.M.S. Palni, Director, G.B. Pant Institute of Himalayan Environment \& Development, Kosi-Katarmal, Almora for providing facilities and encouragements. Sincere thanks to Drs. S.K. Nandi, Scientist-F \& Group Head-BCM \& BTA Group and Dr. R.S. Rawal, Scientist-E, BCM Theme, G.B. Pant Institute of Himalayan Environment \& Development, Kosi-Katarmal, Almora for constant support. We also grateful to Dr. Debjyoti Bhattacharyya, Assam University, Silchar for valuable comments and suggestions.

\section{REFERENCES}

[1] C. Linnaeus, "Species Plantarum,” Vol. 1, London, 1753, pp. 392.

[2] D. J. Mabberley, "Mabberley’s Plant-Book. A Portable Dictionary of Plants, Their Classifications and Uses," 2nd Edition, Cambridge University Press, Cambridge, 2008.

[3] U. C. Pradhan, "A Preliminary enumeration of Rhododendrons of the Indian region-Part 1," Himalayan Plant Journal, Vol. 3, No. 8, 1985, pp. 110-123.

[4] J. Cullen, “A Revision of Rhododendron. I. Subgenus Rhododendron Sections Rhododendron and Pogonanthum," Notes from the Royal Botanic Garden Edinburgh Vol. 39, 1980, pp. 1-207.

[5] D. F. Chamberlain, "A Revision of Rhododendron II. Subgenus Hymenanthes,” Notes from the Royal Botanic Garden Edinburgh, Vol. 39, 1982, pp. 209-486.

[6] W. R. Philipson and M. N. Philipson, “A Revision of Rhododendron III. Subgenera Azaleastrum, Mumeazalea, Candidastrum and Therorhodion," Notes from the Royal Botanic Garden Edinburgh, Vol. 44, 1986, pp. 1-23.

[7] D.F. Chamberlain and S. J. Rae, "A revision of Rhododendron IV. Subgenus Tsutsusi,” Edinburgh Journal of Botany, Vol. 47, 1990, pp. 89-200.

[8] K. A. Kron, "A Revision of Rhododendron Section Pentanthera,” Edinburgh Journal of Botany, Vol. 50, 1993, pp. 249-364.

[9] W. S. Judd and K. A. Kron, “A Revision of Rhododendron VI. Subgenus Pentanthera (Sections Sciadorhodion, Rhodora, and Viscidula)," Edinburgh Journal of Botany, Vol. 52, 1995, pp. 1-54.

[10] U.C. Pradhan, "A Preliminary Enumeration of Rhododendrons of the Indian region-Part 2,” Himalayan Plant Journal, Vol. 4, No. 11-12, 1986, pp. 73-76.

[11] R.B. Ghosh and U.P. Samaddar, "The Rhododendrons of the North-East India," Journal of Economic and Taxonomic Botany, Vol. 13, No. 1, 1989, pp. 205-220.
[12] D. Bhattacharyya and M. Sanjappa, "Rhododendrons Habitats in India," Journal of American Rhododendron Society Vol. 62, No. 1, 2008, pp. 14-18.

[13] A. R. K. Sastry and P.K. Hajra, "Rare and endemic species of Rhododendrons in India-A Preliminary Study," In: S. K. Jain and R. R. Rao, Eds., An Assessment of Threatened Plants of India, BSI, Calcutta, 1983, pp. 222-231.

[14] A. A. Mao, K. P. Singh and P. K. Hajra, "Rhododendrons,” In: N. P. Singh and D. K. Singh, Eds., Floristic Diversity and Conservation Strategies in India, BSI, Calcutta, 2002, pp. 2167-2202.

[15] U. C. Pradhan and S. T. Lachungpa, "Sikkim Himalayan Rhododendrons,” Primulaceae Books, Kalimpong, 1990.

[16] K. K. Singh, S. Kumar, L. K. Rai and A. P. Krishna, "Rhododendron Conservation in Sikkim Himalaya," Current Science, Vol. 85, 2003, pp. 602-606.

[17] J. Y. Yumnam, "Rhododendrons in Meghalaya Need Attention,” Current Science, Vol. 95, No. 7, 2008, pp. 817818.

[18] D. K. Singh and P. K. Hajra, "Floristic Diversity,” In: G. S. Gujral and V. Sharma, Eds., Changing Perspectives of Biodiversity Status in the Himalaya, British Council, New Delhi, 1996.

[19] S. S. Samant, U. Dhar and L. M. S. Palni, "Medicinal Plants of Indian Himalaya: Diversity Distribution Potential Values,” Gyanodaya Prakashan, Nainitial, 1998.

[20] N. P. Singh, J. R. Sharma, K. P. Singh and V. Mudgal, "Species Diversity in Angiosperms," In: N. P. Singh and D. K. Singh, Eds., Floristic Diversity and Conservation Strategies in India, Botanical Survey of India, Kolkata, Vol. 5, 2002, pp. 1631-1674.

[21] Anonymous, "IUCN Red List of Threatened Species," Version 2009.2. http://www.iucnrelist.org/

[22] F. Mingyuan, F. Ruizheng, H. Mingyou, H. Linzhen, Y. Hanbi and D. F. Chamberlain, "Rhododendron," In: W. Zhengyi, P. H. Raven and H. Deyuan, Eds., Flora of China, Science Press, Beijing and Missouri Botanical Garden Press, St. Louis. 2005, Vol. 14, pp. 260-455.

[23] P. Thakur, Y. D. Sharma, B. Kashyap and A. Thakur, "Vegetative propagation of native ornamentals of Himachal Pradesh in India," Abstract of XXVII International Horticultural Congress-IHC2006, Himachal Pradesh, 2006.

[24] K. K. Singh, S. Kumar and R. Shanti, "Raising Planting Materials of Sikkim Himalayan Rhododendron through Vegetative Propagation Using Air-Wet Technique,” Journal of American Rhododendron Society, Vol. 62, 2008, pp. 136-138.

[25] K. K. Singh, and B. Gurung, "In vitro Propagation of $R$. maddeni Hook. f. an Endangered Rhododendron Species of Sikkim Himalaya," Notulae Botanicae Horti Agrobotanici Cluj-Napoca, Vol. 37, No. 1, 2009, pp. 79-83.

[26] G. Lloyd and B. McCown, "Community-Feasible Micropropagation of Mountain Laurel, Kalmia latifolia, by Use of Shoot Tip Culture,” Proceedings of International Plant 
Propagation Society, Vol. 30, 1981, pp. 421-427.

[27] W. C. Anderson, "A revised Medium for Shoot Proliferation of Rhododendron," Journal of American Society \& Horticultural Science, Vol. 109, 1984, pp. 343-347.

[28] G. C. Douglas, "Propagation of Eight Cultivars of Rhododendron in vitro Using Agar-Solidified and Liquid Media and Direct Rooting of Shoots in vivo," Scientia Horticulturae, Vol. 24, 1984, pp. 337-347.

[29] B.H. McCown and G.B. Lloyd, "A Survey of the Response of Rhododendrons to in vitro Cultures," Plant cell tissue and organ culture, Vol. 2, 1985, pp. 77-85.
[30] B. A. Briggs, S. M. McCulloch and L. A. Caton, "In vitro propagation of Rhododendron," Acta Horticulturae, Vol. 364, 1994, pp. 21-26.

[31] P. W. Evers, J. Donkers, A. Prat and E. Vermeer, "Micropropagation of forest trees through tissue culture," Centre for Agricultural Publishing and Documentation, Wageningen, 1988.

[32] R. Almeida, S. Gonçalves and A. Romano, "In vitro Micropropagation of Endangered Rhododendron ponticum L. subsp. baeticum (Boissier \& Reuter) Handel-Mazzetti,” Biodiversity and Conservation Vol. 14, No. 5, 2005, pp. 1059-1069. 